\title{
Ciprofloxacin residue and their impact on biomolecules in eggs of laying hens following oral administration
}

\author{
Md. Mustahsan Billah ${ }^{1,2^{*}}$, S. M. Masud Rana ${ }^{1}$, Mohammad Salim Hossain ${ }^{1 *}$, Sayed Koushik Ahamed ${ }^{3}$, Sujan Banik \\ and Moynul Hasan ${ }^{4}$
}

\begin{abstract}
Background: The present study was designed to evaluate Ciprofloxacin residue and their impact on some biomolecules (albumin, total protein and cholesterol) in eggs of laying hens after oral administration. For that purpose, One group (A) of laying hens $(n=20)$ were orally administered $10 \mathrm{mg} / \mathrm{kg}$ ciprofloxacin for five consecutive days. The second group $(n=10)$ was untreated controls. Eggs were collected from day one of treatment and up to 25 days after withdrawal of treatment. Egg white and yolk from each egg were separated, and ciprofloxacin residues and biomolecules were analyzed by high-performance liquid chromatography method with fluorescence detection and humalyzer having commercial assay kits respectively.
\end{abstract}

Results: Ciprofloxacin was detectable in egg white on the first day of treatment in higher concentrations $(1755 \mu \mathrm{g} / \mathrm{kg})$ while at lower concentrations $(362 \mu \mathrm{g} / \mathrm{kg})$ in egg yolk. In both medium, concentrations increased during five days treatment period. After withdrawal of treatment, eight days and fourteen days were required to deplete the drug residue below the established LOD in albumen and yolk respectively. On the other hand, cholesterol level increased while albumin and total protein level decreased during treatment period. All these biomolecules returns to their normal level at about seventeenth or eighteenth day from the day of treatment. In all cases, the differences in drug residue concentrations and biomolecules concentrations during treatment and post treatment in egg were found significant.

Conclusion: Based on the time needed for residue to deplete below the LOD, we can estimate that, within twenty days of treatment period, egg contents could contain harmful residue which can deplete the nutritional value of egg and thus could cause severe disease for consumer as well whereas it is safe after that period.

Keywords: Ciprofloxacin, HPLC, Biomolecules, Egg white, Egg yolk

\section{Background}

During the last one decade, poultry industries have developed to enormous scale. Nowadays antibiotics especially fluoroquinolones are used on a large scale in poultry industry to cure or prevent diseases to stimulate growth. Fluoroquinolones constitute an intensifying group of synthetic antibiotics which is widely used in the treatment of infections in both human and veterinary medicine.

\footnotetext{
* Correspondence: mustahsan04pharmacy@gmail.com; pharmasalim@yahoo. com

'Department of Pharmacy, Noakhali Science and Technology University, Noakhali 3814, Bangladesh

Full list of author information is available at the end of the article
}

A number of these drugs have been approved to be administered in broiler chickens for the prophylaxis and treatment of respiratory, renal and digestive infections in different areas of the world (Anderson \& Macgowan 2003; Martinez et al. 2006). Fluoroquinolones are one of the few classes of antimicrobial agents having activity against the full range of pathogens involved in broiler chickens, such as Campylobacter jejuni, Salmonella, Shigella or Escherichia coli etc.

It is almost impossible to yield food of animal origin which is completely free from traces of drugs or chemicals (Campbell 1978). Even if no further drug transfer occurred after the dosing period, hens would still store

\section{至 Springer}


drug residues in egg yolks and white for several days to weeks in future (Donoghue et al. 1997). These residues comprise of the parent compound or compounds derived from the parent drug (or both), including metabolites, conjugates and residues bond to macromolecules. Again, biomolecule parameters in serum and egg may provide valuable information for evaluation of health status of birds and reflect many metabolic alterations of organs and tissues (Rivetz et al. 1977). One experiment showed that Ciprofloxacin has toxic effect on some biomolecule parameter in chicken's serum (Salih 2010). But there is no previous study about how ciprofloxacin effect on biomolecules in egg. Currently, the extensive practice of ciprofloxacin in the poultry industry has become a matter of concern because it has led to the emergence of resistance in Salmonella serovars (Esaki et al. 2004; Zhao et al. 2006; San Marti'n et al. 2008), Campylobacter spp. (Griggs et al. 2005; Humphrey et al. 2005; Luangtongkum et al. 2009) and E. coli (Khan et al. 2005; Lee et al. 2005; Lapierre et al. 2008). Residues contained in human food of animal origin may significantly affect the life and health of human causing many troubles as allergic phenomena, sensitization, teratogenic, carcinogenic effects and antibiotic resistance (Michell et al. 1998). Eight thousand and eleven thousand people were infected with fluoroquinolone resistant camphylobacter from eating chickens in 1998 and 1999 respectively in whole world. This circumstances advances public health concerns regarding reduction in the clinical efficacy of FQs in human medicine (Norstro"m et al. 2006; Skjøt-Rasmussen et al. 2009). This devastating history may have adverse effect on consumer confidence in animal product. Considering the above-mentioned issues and the fact that no depletion studies have been reported for ciprofloxacin along with its effect on biomolecules in egg, our study investigates the presence of ciprofloxacin residues in egg albumen and yolk during and post therapeutic treatment via oral routs and determine how many days/weeks still need for eggs to be contaminated with drug residue after the dosing period (withdrawal period) and determine the selected biomolecules level to reduce the potential unintentional contamination of this important food commodity. The withdrawal period can be also defined with the time required for the residue to reach safe concentration from this study. Since no residual tolerance level has been established for ciprofloxacin in eggs, the present study was observed up to the limit of detection (LOD) of the method.

\section{Methods}

This work was performed in the Poultry Research and Training Center (PRTC), Chittagong Veterinary and Animal Sciences University, Chittagong, Bangladesh.

\section{Animals and samples}

Thirty White Leghorn laying hens were used for this experiment. Hens were maintained from 16 weeks of age in conventional cages with an automated system for temperature, humidity and ventilation control. The birds had ad libitum access to water and no medicated feed. The ration was maintained according to breed standard requirements and was formulated by an avian expert. Experimental animals were kept in conditions in compliance with animal welfare guidelines approved by the Bioethics Committee of the Veterinary Medicine Faculty, Chittagong Veterinary and Animal Sciences University. During the experiment, an avian medicine veterinarian observed the hens.

The animals were randomly allocated into two experimental groups: one group of 20 chickens (A) and one group of 10 chickens (B). Group A was treated with a ciprofloxacin $10 \%$ solution, $10 \mathrm{mg} / \mathrm{kg}^{\mathrm{b}} \mathrm{bw}^{-1}$. Group B was the untreated control group. Animals were treated individually once daily for five successive days. The drug was administered using a gastric catheter to ensure proper ingestion of the dose. From day one of treatment and during 25 days after treatment, eggs from each group were collected and preserved at $4 \pm 1{ }^{\circ} \mathrm{C}$ until chromatographic analysis. Egg white and yolk were analyzed separately.

\section{Chemicals and reagents}

The oral commercial formulation used in the experiment, Ciprofloxacin $10 \%$ solution was purchased from the national market (Neofloxin, Beximco Pharma Limited, Bangladesh). A standard of CIP was supplied by Globe Pharmaceuticals, Bangladesh. The LC-grade acetonitrile was supplied by Fisher Chemicals (NJ, USA). The LCGrade acetic acid, absolute ethanol and hexane were from Merck (Darmstadt, Germany). All other reagents were of analytical reagent grade and were obtained from Merck. Standard solutions of ciprofloxacin were prepared in aqueous $0.03 \mathrm{M} \mathrm{NaOH}$ at $1000 \mu \mathrm{g} / \mathrm{mL}$ and stored at $4 \pm 2{ }^{\circ} \mathrm{C}$ in the dark for no longer than 3 months. Working standard solutions were prepared from the standard solution immediately before extraction.

\section{Analytical process}

Egg white and yolk were separated and homogenized individually. The extraction protocol was based on the one developed by Zeng et al. (2005). Concisely, $1 \mathrm{~g}$ of homogenized sample was placed into a $50-\mathrm{mL}$ polypropylene tube and $4 \mathrm{~mL}$ of an acetic acid/ethanol (1:99) solution was added. Samples were shaken for $5 \mathrm{~min}$, and $500 \mu \mathrm{L}$ of acetonitrile and $4 \mathrm{~mL}$ of the acetic/ethanol solution were added to yolk samples. Then, white and yolk samples were placed for $30 \mathrm{~min}$ in a shaker, and samples were centrifuged for $20 \mathrm{~min}$ at $5000 \mathrm{rpm}$. The upper 
layer was transferred into a $10-\mathrm{mL}$ glass tube and evaporated under nitrogen stream at $40 \pm 2{ }^{\circ} \mathrm{C}$; the residue was dissolved with $500 \mu \mathrm{L}$ of acetonitrile. $2 \mathrm{~mL}$ of hexane was added to the samples and were shaken and sonicated, and the upper layer was discarded. The hexane defeating step was repeated in yolk samples. The lower layer was evaporated under a nitrogen stream and the residue was dissolved with $500 \mu \mathrm{L}$ of mobile phase. Samples were transferred to an eppendorf tube and again centrifuged at $7000 \mathrm{rpm}$ for $10 \mathrm{~min}$. The upper layer was filtered by a Millipore filter to an HPLC vial. Residues were separated by liquid chromatography and identified by fluorescence detection (HPLC-FL). Total protein, albumin in egg white and cholesterol concentration in egg yolk were determined in the Humalyzer, Microlab 200 using commercial assay kits (Biolabo, France).

The LOD of the method was $0.5 \mu \mathrm{g} / \mathrm{kg}$ for ciprofloxacin. To evaluate the repeatability and reproducibility of the method, fortified samples at three levels $(1,10$ and $100 \mu \mathrm{g} / \mathrm{kg}$ ) were prepared by spiking $1.0-\mathrm{g}$ blank egg white or yolk homogenate with $40 \mu \mathrm{L}$ of ciprofloxacin working solution. Six replicated samples at each level were prepared and analysed under the same experimental conditions during the same day and on different days. The mean recoveries were as follows: ciprofloxacin $79 \%$ for egg white and $87 \%$ for egg yolk. The precision of the method expressed as CV (\%) was as follows: ciprofloxacin $17.2 \%$ in egg white and $12.5 \%$ in yolk.

\section{Evaluation of drug residue and biomolecule concentrations in egg white and yolk during treatment and post-treatment}

Eggs were collected from the first day of treatment to quantify ciprofloxacin and biomolecules (albumin, total protein and cholesterol) concentrations in egg white and yolk during treatment and post treatment. Five egg samples from each day of treatment were collected from Group A and B and then ciprofloxacin residue and selected biomolecules concentrations were analyzed. Finally, the depletion of ciprofloxacin in both egg compartments and its effect on biomolecules were evaluated.

\section{Statistical analysis}

Data were stated as mean $\pm \mathrm{SE}$, the data were analyzed by (ANOVA). Significant difference between drug concentrations and biomolecules in both egg compartments during treatment and post treatment were assessed using students' $t$ test. $P<0.05$ was considered as statistically significant (SAS SAS/STAT Users Guide 1989).

\section{Results}

Ciprofloxacin concentrations in egg white and yolk during the experiment are shown in Fig. 1. Ciprofloxacin residues were identified in egg white from the first day of treatment with mean drug concentrations of $1755 \pm$ $197 \mu \mathrm{g} / \mathrm{kg}$ having gradual increase during all the treatment administration period. It is also found that residues in egg white dropped rapidly from the concentration of $2394 \pm 497 \mu \mathrm{g} / \mathrm{kg}$, on the first day following withdrawal of the drug, to $29 \pm 6 \mu \mathrm{g} / \mathrm{kg}$ on the eighth day following withdrawal of the drug, and were not detectable by the thirteenth day after treatment. In egg yolk, ciprofloxacin residue on the first day of treatment $(362 \pm 139 \mu \mathrm{g} / \mathrm{kg})$ was considerably lower than in white. Moreover, ciprofloxacin concentration in yolk constantly increased reaching $962 \pm 218 \mu \mathrm{g} / \mathrm{kg}$ on fifth day of treatment. After withdrawal, the concentration of Ciprofloxacin in yolk continued to increase until day three, when it reached a maximum concentration $(1682 \pm 197 \mu \mathrm{g} / \mathrm{kg})$. On the fourth day after withdrawal, the concentrations in egg yolk started to decrease, and residues were not detectable by the seventeenth day from the day of treatment in any eggs. According to the statistical analysis, the differences of the Ciprofloxacin residue concentrations during treatment and post treatment statistically significant $(P<0.05)$, when compared to control.

In Fig. 2, it was found that cholesterol level in egg yolk increased gradually up to nine days, when it reached the maximum concentration. From tenth day, the concentration decreased constantly up to sixteenth day. After that day, the concentration remained constant and reached to its normal level when compared to controlled group. But in Figs. 3 and 4, it was found that albumin and total protein level in egg white showed just opposite result of the cholesterol level. Both of the biomolecules decreased gradually up to seven days reaching their minimum concentration. From the day of eight, the concentration increased constantly up to sixteenth day. After that day, the concentration remained constant and reached to its normal level in comparison to controlled group. According to the statistical analysis, the differences of the biomolecules concentrations during treatment and post treatment were found statistically significant $(P<0.05)$.

\section{Discussion}

Ciprofloxacin is a major, active metabolite of Enrofloxacin in different species and is formed by the de-ethylation of Enrofloxacin. Ciprofloxacin was the first of the two compounds to be developed and is only approved for use in humans (Tyczkowska et al. 1989). Veterinary drugs can be absorbed by the digestive tract of laying hens and transferred to the egg. Physicochemical characteristics of these compounds determine their pharmacokinetic behavior and distribution to and within egg compartments. Traditionally the quite lipid soluble drugs are expected to yield residues only in fat-rich yolk. However, the quite lipid soluble drug as well as many other drugs-showed higher residues in white than in yolk during long 


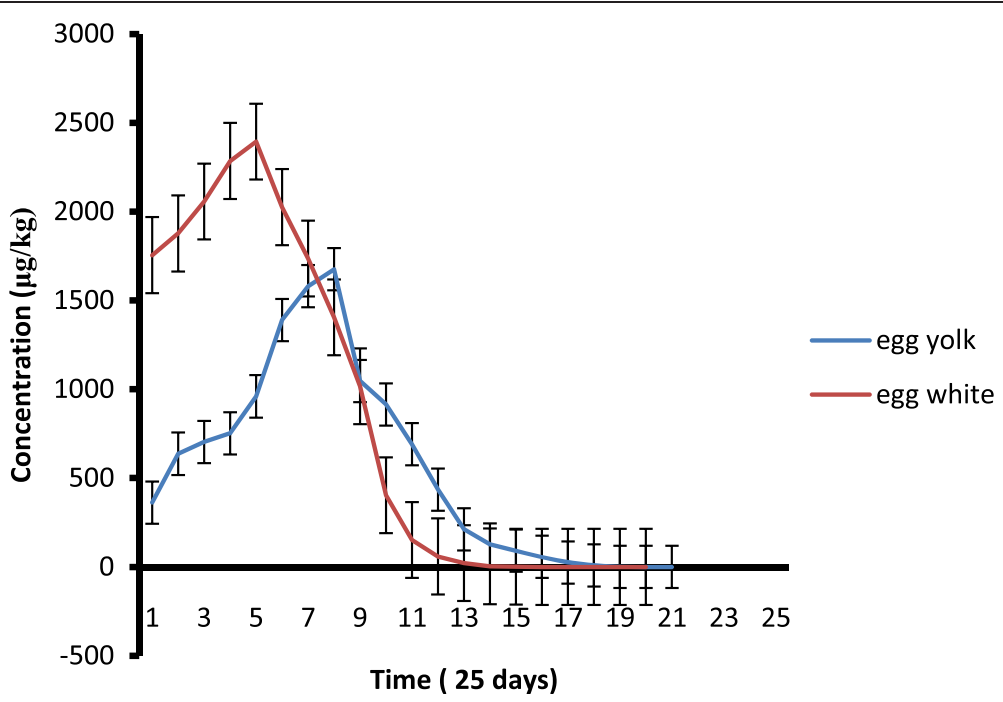

Fig. 1 Ciprofloxacin residues in egg white and yolk from laying hens treated with $10 \mathrm{mg} / \mathrm{kg} . \mathrm{bw}^{-1}$ of ciprofloxacin $10 \%$ solution

term administration (Kan \& Petz 2000). Literature data on the distribution of drugs between egg white and yolk showed a reasonable consistency within drugs (Kan \& Petz 2000). Concern of antimicrobial residues found in meat, milk or eggs after an antibiotic animal treatment are related to the probable induction of allergic reactions in humans and the resistance of human enteric bacteria.

In this experiment, a 5-day treatment was implemented because it is the standard number of days used with fluoroquinolones administration to laying hens. The number of animals for each experimental group was developed to comply with the veterinary guidelines for the harmonization of withdrawal periods. Twenty laying hens were allocated to experimental group A, to obtain at least five eggs per day for the HPLC analysis during the depletion period. Eggs of the untreated control group were used to validate the method and to compare the experimental study. Fluoroquinolone depletion from egg white and yolk can be correlated with the egg formation process. The whole egg formation takes about 12 days. Lipoproteins synthesized in the liver are the main components of the egg yolk. The maturation process finalizes in about 10 days. The egg white is mainly developed by watersoluble proteins secreted by the hen oviduct (Donoghue \& Myers 2000; Kan 2003). The distribution of the drugs between egg yolk and white is determined by the physiologic processes (Kan \& Petz 2000; Donoghue \& Hairston 2000). For this reason, Ciprofloxacin residues were noticed from the first day of treatment in high level average concentrations of over $1100 \mu \mathrm{g} / \mathrm{kg}$.

In this study, Ciprofloxacin residues in egg white remained gradual increase during the 5 days of treatment. In egg yolk, Ciprofloxacin residues were detectable after the first day of treatment but the average

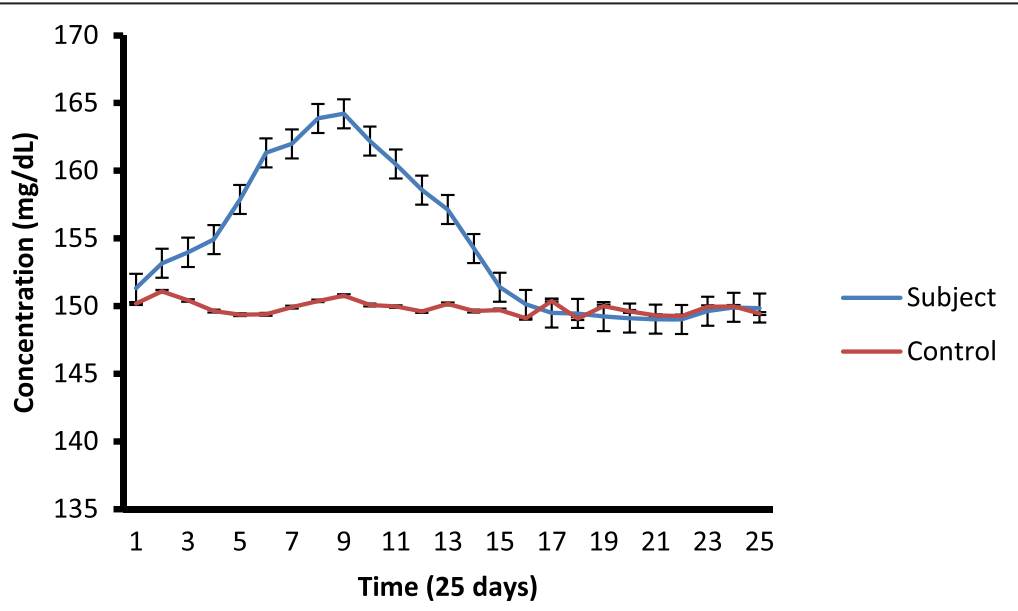

Fig. 2 Cholesterol level in egg yolk from laying hens treated with $10 \mathrm{mg} / \mathrm{kg} . \mathrm{bw}^{-1}$ of ciprofloxacin $10 \%$ solution in comparison with control group (B) 


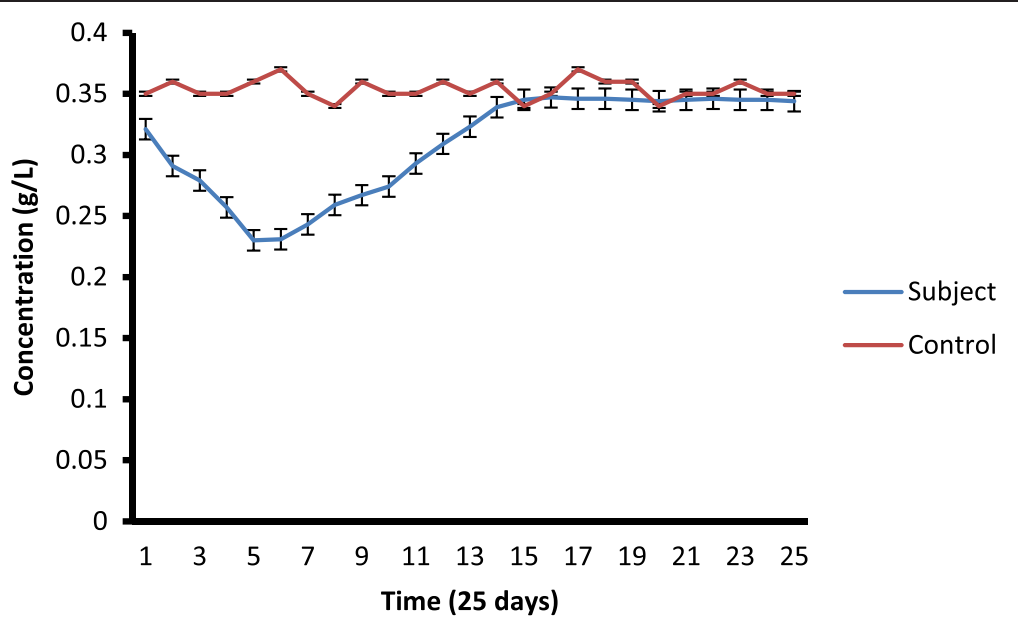

Fig. 3 Albumin level in egg white from laying hens treated with $10 \mathrm{mg} / \mathrm{kg} . \mathrm{bw}^{-1}$ of ciprofloxacin $10 \%$ solution in comparison with control group (B)

concentrations were inferior to white. The residue increased during the 5 days of drug administration (from 362 to $962 \mu \mathrm{g} / \mathrm{kg}$ ). Residues in yolk accumulate during growth of the follicle, and therefore, depending on the length and time of exposure to the drug relative to yolk deposition, drug levels can increase, constant or decrease (Kan 2003). According to Kan and Petz (2000), residues of drugs in yolk generally required exposure for about eight to ten days to reach its constant level. However, Donoghue et al. (1996) describes that a single exposure to a drug might be adequate to detect the drug in either egg white or yolk, depending on the characteristics of the drug and sensitivity of the analytical method applied. Gharieb and Atti (2011) designates that, 5 days of therapeutic treatment resulted a Ciprofloxacin residue in egg white and egg yolk until 6 days post-oral administration. During the depletion study, after drug withdrawal, Ciprofloxacin residues showed different depletion profiles in egg white and yolk. In egg white, Ciprofloxacin residues dropped rapidly from day one to day four after withdrawal and were detectable up to fourteenth day from the day of treatment. In egg yolk, Ciprofloxacin concentrations were lower than in egg white, but they were noticeable until eighteenth day from the day of treatment.

On the base of our findings, we propose that yolk is a better medium than white for monitoring ciprofloxacin residue in eggs. The results obtained for ciprofloxacin were almost similar to previous studies. Cornejo et al. (2011) revealed that, 8 days and 10 days are required for enrofloxacin residues in egg whites and yolk respectively to deplete below the established LOD while 26 days and 20 days are required for flumiquine residues in egg whites and yolk respectively. Lolo et al. (2005) reported that enrofloxacin with ciprofloxacin residues were detectable in both white and yolk for 15 days after drug withdrawal in concentrations of 6.4 and $3.5 \mu \mathrm{g} / \mathrm{kg}$,

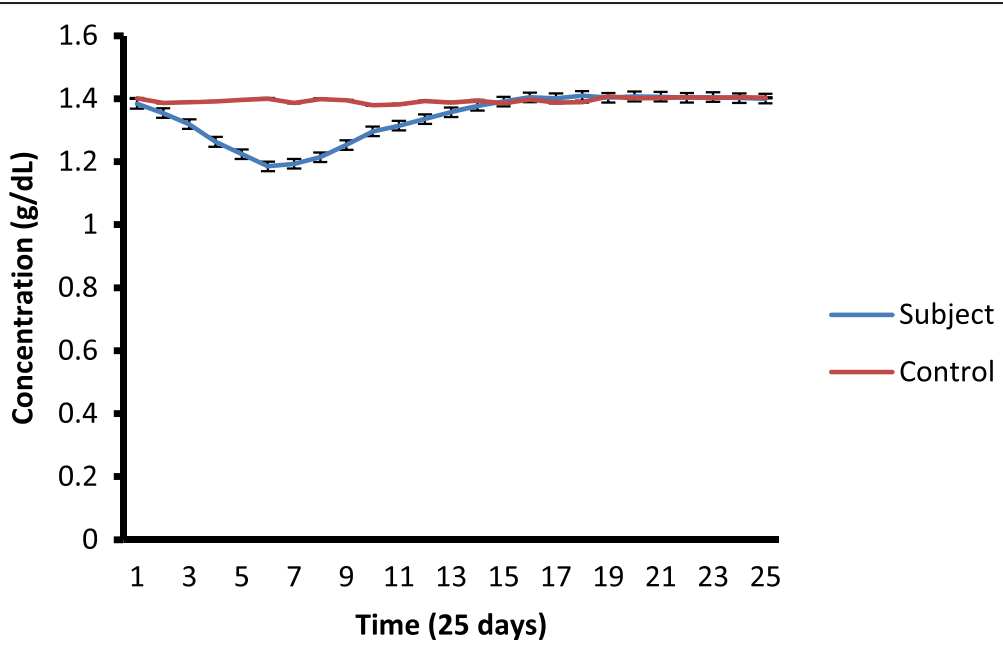

Fig. 4 Total protein level in egg white from laying hens treated with 10 mg/kg.bw ${ }^{-1}$ of ciprofloxacin $10 \%$ solution in comparison with control group (B) 
respectively. Similar studies have been carried out with other fluoroquinolones. Yang et al. (2006) studied danofloxacin depletion in eggs and reported that the residues were detectable up to day four in albumen and up to day eleven in yolk. This depletion profile was longer than the one described by Chu et al. (2002) for total radioactive residues of sarafloxacin, which were detectable for up to 1 day in egg white and for 6 days in egg yolk. According to Kan (2003), the reason for the different distribution in egg white and yolk of the fluoroquinolones may be because of the differences in liposolubility and physicochemical characteristics of the drugs, such as their molecular weight, $\mathrm{pKa}$ value and binding capacity to plasma proteins (Kan \& Petz 2000). Although there is no universal agreement regarding fluoroquinolone distribution, some researchers defined that these drugs accumulate mainly in the yolk, being deposited and combined to this matrix during the egg development (Donoghue \& Hairston 2000). In another study, Donoghue and Myers (2000) showed that drugs can be fused in developing egg yolks, even after a single dose. However, other researchers (Kan 2003; Donoghue et al. 1996; Riberzani et al. 1993; Gorla et al. 1997; Roudaut 1998) suggested that fluoroquinolone residue content is higher in egg white.

Following the depletion study, we tried to find out the effect of Ciprofloxacin on the selected biomolecules (albumin, total protein and cholesterol) in egg during the whole experimental period. Cholesterol level continued increase constantly even after withdrawal period, up to four days from the day of treatment, while albumin and total protein level remained decrease constantly even after withdrawal period, up to two days from the day of treatment. This findings is similar to another study, where the significant effect of ciprofloxacin for twenty days of experimental period on some biomolecules in blood serum of chickens was found (Salih 2010). Our findings prove that there is significant correlation between the drug residue and biomolecules concentration in egg.

The high levels of Ciprofloxacin residues in egg white and yolk can involve some risks to human health such as adverse effects on bacterial flora in the human gastrointestinal tract, and the emergence and dissemination of resistant bacteria (Anderson \& Macgowan 2003; Liu et al. 2005; Nelson et al. 2007). Again, the high level of cholesterol in yolk may cause severe cardiovascular disease. Therefore, the egg withdrawal time following Ciprofloxacin administration to laying hens should be established by regulatory authorities to minimize the risks associated with consumption of fluoroquinolones. Moreover, before any drug can be marketed for use in food-producing animals, three significant considerations must be addressed: 1) identify and measuring residues in edible tissues 2) determining from toxicity tests the conditions for the safe use of the drug with respect to the persistence of the residues 3) assuring in the use that residues don't exceed the amount which is regarded as safe.

\section{Conclusion}

Our present study showed that almost ten days is required for Ciprofloxacin residues in egg whites to deplete below the established LOD $(0.5 \mu \mathrm{g} / \mathrm{kg})$ and fourteen days in egg yolks. It also demonstrated that cholesterol, albumin and total protein level become normal concentration after twenty days from the beginning of treatment. Considering that no MRL have been established for ciprofloxacin in eggs and its effect on biomolecules, we recommend egg withdrawal times of fifteen days after 5-days administration of Ciprofloxacin in legislation the whole egg is considered and not separately the egg yolk and the egg white. We also suggest that, egg yolk is the ideal matrix for ciprofloxacin residue monitoring in eggs and after twenty days from the day of treatment, ciprofloxacin residue depletes to its undetectable level and biomolecules returns to their normal concentration. So, within twenty days of treatment period, egg contents could contain harmful residue which can deplete the nutritional value of egg and thus could cause severe disease for consumer while it is safe after that period. Hence, further research is desired to find out any effect of Ciprofloxacin on other biomolecules in eggs following other route of administration.

Abbreviations
HPLC: High Performance Liquid Chromatography; LC: Liquid Chromatography;
LOD: Limit of Detection; PRTC: Poultry Research and Training Center.

Competing interests

The authors declare that they have no competing interests.

\section{Authors' contribution}

MMB- participated in experiments, study design, manuscript preparation. SMMR- participated in statistics analysis. MSH-Supervising and directing the project. SKA, SB, MH-checked the grammatical mistakes and corrected the final manuscript. All authors read and approved the final version of the manuscript.

\section{Acknowledgement}

The Authors thank the Department of Physiology, Biochemistry and Pharmacology, Faculty of Veterinary Medicine, Chittagong Veterinary and Animal Sciences University, Chittagong, Bangladesh for their valuable support, suggestions and encouragement during the research work. The authors also thank Globe Pharmaceuticals, Noakhali, Bangladesh for providing Ciprofloxacin standard for this research purpose.

\section{Author details}

'Department of Pharmacy, Noakhali Science and Technology University, Noakhali 3814, Bangladesh. ${ }^{2}$ Department of Pharmacy, Dhaka International University, Dhaka 1207, Bangladesh. ${ }^{3}$ Department of Pharmacy, Comilla University, Comilla 3506, Bangladesh. ${ }^{4}$ Department of Pharmacy, Jagannath University, Dhaka-4 3506, Bangladesh.

Received: 19 July 2015 Accepted: 15 October 2015

Published online: 05 November 2015 


\section{References}

Anderson M, Macgowan A. Development of the quinolones. J Antimicrob Chemother. 2003;51:1-11.

Campbell DJ. Drug residue in animal tissues and their regulatory significance the Canadian point of view. J AOAC. 1978;61(5):1194-7.

Chu P, Wang R, Chu H. Liquid chromatographic determination of fluoroquinolones in egg albumen and egg yolk of laying hens using fluorometric detection. J Agric Food Chem. 2002;50:4452-5.

Cornejo J, Lapierre L, Iragu“" en D, Cornejo S, Cassus G, Richter P, et al. Study of enrofloxacin and flumequine residues depletion in eggs of laying hens after oral administration. J Vet Pharmacol Ther. 2011;35:67-72.

Donoghue DJ, Hairston $\mathrm{H}$. Food safety implication: certain antibiotics may rapidly contaminate egg albumen during the process of its formation. Br Poult Sci. 2000;41:174-7.

Donoghue D, Myers K. Imaging residue transfers into egg yolks. J Agric Food Chem. 2000;48:6428-30.

Donoghue DJ, Hairston H, Gaines S, Bartholmew MJ, Donoghue AM. Modeling residue uptake in eggs: similar drug residue patterns in developing yolks following injection with ampicillin or oxytetracycline. Poult Sci. 1996;75:321-8.

Donoghue DJ, Hairston H, Podhorniak LV. Modeling drug residue uptake by eggs: evidence of a consistent daily pattern of contaminant transfer into developing preovulatory yolks. J Food Prot. 1997;60:1251-5.

Esaki H, Morioka A, Ishihara K, Kojima A, Shiroki A, Tamura Y, et al. Antimicrobial susceptibility of Salmonella isolated from cattle, swine and poultry (2001-2002): report from the Japanese Veterinary Antimicrobial Resistance Monitoring Program. J Antimicrob Chemother. 2004;53:266-70.

Gharieb MM, Atti NMA. Ciprofloxacin drug residue in table egg. SCVMJ. 2011;16(2):137-8

Gorla N, Chiostri E, Ugnia L, Weyers A, Giacomelli N, Davicino R, et al. HPLC residues of enrofloxacin and ciprofloxacin in eggs of laying hens. Int J Antimicrob Agents. 1997;9:253-6.

Griggs D, Johnson M, Frost J, Humphrey T, Jørgensen F, Piddock L. Incidence and mechanism of ciprofloxacin resistance in Campylobacter spp. isolated from commercial poultry flocks in the United Kingdom before, during, and after fluoroquinolone Treatment. Antimicrob Agents Chemother. 2005;49:699-707.

Humphrey TJ, Jørgensen F, Frost J, Wadda H, Domingue G, Elviss N. Prevalence and subtypes of ciprofloxacin-resistant Campylobacter spp. in commercial poultry flocks before, during, and after treatment with fluoroquinolones. Antimicrob Agents Chemother. 2005;49:690-8.

Kan C. Residues of Veterinary Drugs in Eggs and their Distribution Between Yolk and White. Alemania: Wuppertal University; 2003.

Kan C, Petz M. Residues of veterinary drugs in eggs and their distribution between yolk and white. J Agric Food Chem. 2000;48:6397-403.

Khan AA, Nawaz MS, SummageWest C, Khan SA, Lin J. Isolation and molecular characterization of fluoroquinolone-resistant Escherichia coli from poultry litter. Poult Sci. 2005;84:61-6.

Lapierre L, Cornejo J, Borie C, Toro C, San Martı'n B. Genetic characterization of antibiotic resistance genes linked to class 1 and class 2 integrons in commensal strains of Escherichia coli isolated from poultry and swine. Microb Drug Resist. 2008;14:265-72.

Lee YJ, Cho JK, Kim KS, Tak RB, Kim AR, Kim JW, et al. Fluoroquinolone resistance and gyrA and parC mutations of Escherichia coli isolated from Chicken. J Microbiol. 2005;43:391-7.

Liu JH, Chen ZL, Li Y, Liu YW. Effects of low concentration enrofloxacin on SPF mice intestinal microflora. Sci Agric Sin. 2005;38:1905-10.

Lolo M, Pedreira S, Fente C, Vaa"zquez B, Franco C, Cepeda A. Study of enrofloxacin depletion in the eggs of laying hens using diphasic dialysis extraction / purification and determinative HPLC-MS analysis. J Agric Food Chem. 2005;53:2849-52.

Luangtongkum T, Jeon B, Han J, Plummer P, Logue CM, Zhang Q. Antibiotic resistance in Campylobacter: emergence, transmission and persistence. Future Microbiol. 2009;2:189-200.

Martinez M, McDermott P, Walker R. Pharmacology of the Fluoroquinolones: a perspective for the use in domestic animals. Vet J. 2006;172:10-28.

Michell JM, Griffiths MW, McEwen SA, Mcab W, Yee AJ. Antimicrobial drug residues in milk and meat: causes, concerns, prevalence, regulations, tests and test performance. J Food Prot. 1998;61(6):742-56.

Nelson JM, Chiller TM, Powers JH, Angulo FJ. Fluoroquinolone- resistant Campylobacter species and the withdrawal of fluoroquinolones from use in poultry: a public health success story. Clin Infect Dis. 2007;44:977-80.
Norstro“m M, Hofshagen M, Stavnes T, Schau J, Lassen J, Kruse H. Antimicrobial resistance in Campylobacter jejuni from humans and broilers in Norway. Epidemiol Infect. 2006;134:127-30.

Riberzani A, Fedrizzi G, Espositi S. Presence of flumequine in eggs: experimental results of a simulated field trial. Euroresidue. 1993;2:576-80.

Rivetz B, Begin E, Hornstein K, Nerdinger M. Biochemical changes in fowl serum during infection with strains of Newcastle disease virus of different virulence. Changes in serum proteins, uni acid, lipids and electrolytes. Res Vet Sci. 1977;22:285-91.

Roudaut B. Elimination of oxolinic acid in eggs after oral treatment of laying hens. Br Poult Sci. 1998;39:47-52

Salih YZ. Toxic effect of ciprofloxacin on some biochemical variables in chickens. Iraqi J Vet Sci. 2010;24(2):137.

San Martı'n B, Lapierre L, Cornejo J, Bucarey S. Characterization of antibiotic resistance genes linked to class 1 and 2 integrons in strains of Salmonella spp. isolated from swine. Can J Microbiol. 2008;54:569-76.

SAS SAS / STAT Users Guide. Ver 6. SAS Institute Inc. Cary, NC. 1989.

Skjøt-Rasmussen L, Ethelberg S, Emborg HD, Agersø Y, Larsen LS, Nordentoft S, et al. Trends in occurrence of antimicrobial resistance in Campylobacter jejuni isolates from broiler chickens, broiler chicken meat, and human domestically acquired cases and travel associated cases in Denmark. Int J Food Microbiol. 2009;131:277-9

Tyczkowska K, Hedeen KM, Aucoin DP, Aronson AL. High-performance liquid chromatographic method for the simultaneous determination of enrofloxacin and its primary metabolite ciprofloxacin in canine serum and prostatic tissue. J Chromatogr. 1989;493:337-46.

Yang G, Dong A, Zeng Z, Huang X, Chen Z. Study of danofloxacin depletion in eggs of laying hens after oral administration. Int J Antimicrob Agents. 2006;28:128-31.

Zeng Z, Dong A, Yang G, Chen Z, Huang X. Simultaneous determination of nine fluoroquinolones in egg white and egg yolk by liquid chromatography with fluorescence detection. J Chromatogr B Anal Technol Biomed Life Sci. 2005;821:202-9

Zhao S, Mcdermott PF, Friedman S, Abbott J, Ayers S, Glenn A, et al. Antimicrobial resistance and genetic relatedness among Salmonella from retail foods of animal origin: NARMS retail meat surveillance. Foodborne Pathog Dis. 2006;3:106-17.

\section{Submit your manuscript to a SpringerOpen ${ }^{\circ}$ journal and benefit from:}

- Convenient online submission

- Rigorous peer review

- Immediate publication on acceptance

- Open access: articles freely available online

- High visibility within the field

- Retaining the copyright to your article

Submit your next manuscript at springeropen.com 